\title{
Metric stability of trees and tight spans
}

\section{Journal Article}

Author(s):

Lang, Urs; Pavón, Maël; Züst, Roger

Publication date:

2013-07

Permanent link:

https://doi.org/10.3929/ethz-b-000070297

Rights / license:

In Copyright - Non-Commercial Use Permitted

Originally published in:

Archiv der Mathematik = Archives of mathematics = Archives mathématiques 101(1), https://doi.org/10.1007/s00013-013-0535-y 


\title{
Metric stability of trees and tight spans
}

\author{
Urs Lang, Mä̈l Pavón, And Roger Züst
}

\begin{abstract}
We prove optimal extension results for roughly isometric relations between metric $(\mathbb{R}$-)trees and injective metric spaces. This yields sharp stability estimates, in terms of the Gromov-Hausdorff (GH) distance, for certain metric spanning constructions: the GH distance of two metric trees spanned by some subsets is smaller than or equal to the $\mathrm{GH}$ distance of these sets. The GH distance of the injective hulls, or tight spans, of two metric spaces is at most twice the GH distance between themselves.
\end{abstract}

Mathematics Subject Classification (2010). 51K.

Keywords. Gromov-Hausdorff distance, Injective hull, Tight span, Metric tree.

1. Introduction. The main purpose of this note is to provide an optimal stability result, in terms of the Gromov-Hausdorff distance, for Isbell's [8] injective hull construction $X \mapsto \mathrm{E}(X)$ for metric spaces. Roughly speaking, $\mathrm{E}(X)$ is a smallest injective metric space containing an isometric copy of $X$ (all relevant definitions will be reviewed later in this paper). Here, a metric space $Y$ is called injective if for any isometric embedding $i: A \rightarrow B$ of metric spaces and any 1-Lipschitz (i.e., distance nonincreasing) map $f: A \rightarrow Y$ there exists a 1-Lipschitz extension $g: B \rightarrow Y$ of $f$, so that $g \circ i=f$ (see [1, Section 9] for the general categorical notion). Examples of injective metric spaces include the real line $\mathbb{R}, l_{\infty}(I)$ for any index set $I$, and all complete metric trees; however, by Isbell's result, this list is by far not exhaustive. Injective metric spaces are complete, geodesic, and contractible and share a number of remarkable properties. We refer to [9, Sections 2 and 3] for a recent survey of injective metric spaces and hulls.

Research supported by the Swiss National Science Foundation. 

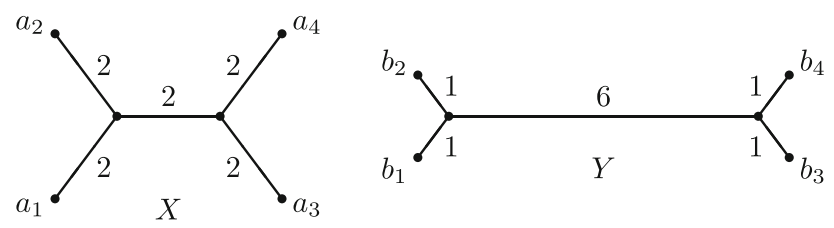

FiguRE 1. Two matric trees $x$ and $y$ with $d_{G H(x, y)}=1$

An alternative, but equivalent, description of $\mathrm{E}(X)$ was given later by Dress [5], who called it the tight span of $X$. If $X$ is compact, then so is $\mathrm{E}(X)$, and if $X$ is finite, $\mathrm{E}(X)$ has the structure of a finite polyhedral complex of dimension at most $|X| / 2$ with cells isometric to polytopes in some finitedimensional $l_{\infty}$ space. If every quadruple of points in $X$ admits an isometric embedding into some metric tree, then so does $X$ itself, and $\mathrm{E}(X)$ provides the minimal complete such tree for $X$. This last property makes the injective hull/tight span construction a useful tool in phylogenetic analysis. Based on genomic differences an evolutionary distance between similar species is defined, and the construction may then be applied to this finite metric space. Due to noise in the measurements or systematic errors, the process will rarely yield a tree, but (the 1-skeleton of) the resulting polyhedral complex may still give a good indication on the phylogenetic tree one tries to reconstruct (compare $[6,7]$ and the references there).

In view of these applications, and also from a purely geometric perspective, it is interesting to know how strongly the injective hull is affected by small changes of the underlying metric space. The dissimilarity of two metric spaces $A, B$ is conveniently measured by their Gromov-Hausdorff distance $d_{\mathrm{GH}}(A, B)$. Moezzi [10, Theorem 1.55] observed that $d_{\mathrm{GH}}(\mathrm{E}(A), \mathrm{E}(B))$ is not larger than eight times $d_{\mathrm{GH}}(A, B)$. Here it is now shown that in fact

$$
d_{\mathrm{GH}}(\mathrm{E}(A), \mathrm{E}(B)) \leq 2 d_{\mathrm{GH}}(A, B),
$$

and an example is constructed to demonstrate that the factor two is optimal (see Section 3). Furthermore, we prove that if both $\mathrm{E}(A)$ and $\mathrm{E}(B)$ are metric trees (in the most general sense of $\mathbb{R}$-trees), then

$$
d_{\mathrm{GH}}(\mathrm{E}(A), \mathrm{E}(B)) \leq d_{\mathrm{GH}}(A, B),
$$

without a factor two. In particular, if $X$ and $Y$ are finite simplicial metric trees with sets of terminal vertices $A$ and $B$, respectively, then $d_{\mathrm{GH}}(X, Y) \leq$ $d_{\mathrm{GH}}(A, B)$. This result (which we have not been able to find in the literature) is not as obvious as it may appear at first glance. A complication arises from the fact that for the respective vertex sets $V_{X}, V_{Y}$, it is not true in general that $d_{\mathrm{GH}}\left(V_{X}, V_{Y}\right) \leq d_{\mathrm{GH}}(A, B)$, not even for combinatorially equivalent binary trees. For instance, consider the two trees $X, Y$ depicted in Fig. 1, with the indicated edge lengths. The correspondence between $A:=\left\{a_{1}, \ldots, a_{4}\right\}$ and $B:=\left\{b_{1}, \ldots, b_{4}\right\}$ that relates $a_{i}$ to $b_{i}$ distorts all distances by an additive error of two. Since the diameters of $A$ and $B$ also differ by two, no correspondence (i.e., left- and right-total relation) between $A$ and $B$ has (maximal) distortion 
less than two. The Gromov-Hausdorff distance equals one half this minimal number (see Section 3 ), so $d_{\mathrm{GH}}(A, B)=1$. Similar considerations show that $d_{\mathrm{GH}}\left(V_{X}, V_{Y}\right)=2$. Yet, $d_{\mathrm{GH}}(X, Y)=1$. For the proof, points in $X$ and $Y$ need to be related in a non-canonical way.

2. Extension of roughly isometric relations. As just indicated, the GromovHausdorff distance may be characterized in terms of the additive distortion of relations between the two given metric spaces. Therefore, in this section, we begin by studying the possibility of extending relations without increasing the distortion.

Let $X, Y$ be two metric spaces. We write $\left|x x^{\prime}\right|$ for the distance of two points $x, x^{\prime} \in X$ and, likewise, $\left|y y^{\prime}\right|$ for the distance of $y, y^{\prime} \in Y$. Given a relation $R$ between $X$ and $Y$, i.e., a subset of $X \times Y$, the distortion of $R$ is defined as the (possibly infinite) number

$$
\operatorname{dis}(R):=\sup \left\{|| x x^{\prime}|-| y y^{\prime}||:(x, y),\left(x^{\prime}, y^{\prime}\right) \in R\right\} .
$$

In case $R$ is given by a map $f: X \rightarrow Y$, we write $\operatorname{dis}(f)$ for $\operatorname{dis}(R)$. If $\operatorname{dis}(f) \leq \varepsilon$ for some $\varepsilon \geq 0$, then $f$ is called $\varepsilon$-roughly isometric. This means that

$$
\left|x x^{\prime}\right|-\varepsilon \leq\left|f(x) f\left(x^{\prime}\right)\right| \leq\left|x x^{\prime}\right|+\varepsilon
$$

for every pair of points $x, x^{\prime} \in X$. See [3, Chapter 7] and [4, Chapter 7] for this terminology. We denote by $\pi_{X}: X \times Y \rightarrow X$ and $\pi_{Y}: X \times Y \rightarrow Y$ the canonical projections. For a set $A \subset X$, we say that $A$ spans $X$ if, for every pair $\left(x, x^{\prime}\right) \in X \times X$,

$$
\left|x x^{\prime}\right|=\sup _{a \in A}\left(|x a|-\left|x^{\prime} a\right|\right)
$$

equivalently, for all $\varepsilon>0$ there is an $a_{\varepsilon} \in A$ such that $\left|x x^{\prime}\right|+\left|x^{\prime} a_{\varepsilon}\right| \leq\left|x a_{\varepsilon}\right|+\varepsilon$. The definition is motivated by the fact that the injective hull of a metric space $A$ may be characterized as an injective metric extension $X \supset A$ spanned by $A$, see Proposition 3.3 below. For a constant $\alpha \geq 0$, a set $S \subset X$ is called an $\alpha$-net in $X$ if for every $x \in X$ there exists a $z \in S$ such that $|x z| \leq \alpha$.

Proposition 2.1. Suppose that $X, Y$ are two injective metric spaces. If $R \subset$ $X \times Y$ is a set with $\alpha:=\operatorname{dis}(R) / 2<\infty$ and the property that $\pi_{X}(R)$ spans $X$, there exists an extension $R \subset \bar{R} \subset X \times Y$ such that $\pi_{X}(\bar{R})$ is an $\alpha$-net in $X$ and $\operatorname{dis}(\bar{R})=\operatorname{dis}(R)$.

In particular, every $\varepsilon$-roughly isometric map $f: A \rightarrow Y$ defined on a set $A \subset X$ that spans $X$ admits an $\varepsilon$-roughly isometric extension $\bar{f}: S \rightarrow Y$ to some $\varepsilon / 2$-net $S$ in $X$ and, hence, also a $2 \varepsilon$-roughly isometric extension $\hat{f}: X \rightarrow Y$. Below we shall use the simple fact that every injective metric space $Y$ is hyperconvex [2] (the converse is true as well). This means that for every family $\left\{\left(y_{i}, r_{i}\right)\right\}_{i \in I}$ in $Y \times \mathbb{R}$ with the property that $r_{i}+r_{j} \geq\left|y_{i} y_{j}\right|$ for all pairs of indices $i, j \in I$, there is a point $y \in Y$ such that $\left|y y_{i}\right| \leq r_{i}$ for all $i \in I$. 
Proof. It suffices to show that for every set $R \subset X \times Y$ with $\alpha:=\operatorname{dis}(R) / 2<\infty$ and the property that $\pi_{X}(R)$ spans $X$ and for every $\bar{x} \in X$ there exists a pair $\left(x_{0}, y_{0}\right) \in X \times Y$ such that $\left|\bar{x} x_{0}\right| \leq \alpha$ and

$$
\operatorname{dis}\left(R \cup\left\{\left(x_{0}, y_{0}\right)\right\}\right)=\operatorname{dis}(R) .
$$

The general result then follows by an application of Zorn's lemma.

Thus let such $R$ and $\bar{x}$ be given, and put $\alpha:=\operatorname{dis}(R) / 2$. For all $(x, y)$, $\left(x^{\prime}, y^{\prime}\right) \in R$,

$$
|| x x^{\prime}|-| y y^{\prime}|| \leq 2 \alpha
$$

and $(|x \bar{x}|+\alpha)+\left(\left|x^{\prime} \bar{x}\right|+\alpha\right) \geq\left|x x^{\prime}\right|+2 \alpha \geq\left|y y^{\prime}\right|$. Hence, since $Y$ is hyperconvex, there is a point $y_{0} \in Y$ such that for all $(x, y) \in R$,

$$
\left|y y_{0}\right| \leq|x \bar{x}|+\alpha .
$$

Furthermore, since $\pi_{X}(R)$ spans $X$, for every $(x, y) \in R$ and $\varepsilon>0$ there exists $\left(x_{\varepsilon}, y_{\varepsilon}\right) \in R$ such that $|x \bar{x}|+\left|\bar{x} x_{\varepsilon}\right| \leq\left|x x_{\varepsilon}\right|+\varepsilon$ and, hence,

$$
\left|y y_{0}\right| \geq\left|y y_{\varepsilon}\right|-\left|y_{0} y_{\varepsilon}\right| \geq\left(\left|x x_{\varepsilon}\right|-2 \alpha\right)-\left(\left|\bar{x} x_{\varepsilon}\right|+\alpha\right) \geq|x \bar{x}|-3 \alpha-\varepsilon .
$$

Since this holds for all $\varepsilon>0$, it follows that $\left|y y_{0}\right| \geq|x \bar{x}|-3 \alpha$. For every $(x, y) \in R$, put $r(x, y):=\left|y y_{0}\right|+2 \alpha$, and set $r(\bar{x}):=\alpha$. We have $r(x, y)+r(\bar{x})=$ $\left|y y_{0}\right|+3 \alpha \geq|x \bar{x}|$ and $r(x, y)+r\left(x^{\prime}, y^{\prime}\right) \geq\left|y y^{\prime}\right|+4 \alpha \geq\left|x x^{\prime}\right|+2 \alpha \geq\left|x x^{\prime}\right|$, for all $(x, y),\left(x^{\prime}, y^{\prime}\right) \in R$. Thus, since $X$ is hyperconvex, there exists a point $x_{0} \in X$ such that

$$
\left|x x_{0}\right| \leq r(x, y)=\left|y y_{0}\right|+2 \alpha
$$

and $\left|\bar{x} x_{0}\right| \leq r(\bar{x})=\alpha$ for all $(x, y) \in R$. Then also

$$
\left|y y_{0}\right| \leq|x \bar{x}|+\alpha \leq\left|x x_{0}\right|+\left|\bar{x} x_{0}\right|+\alpha \leq\left|x x_{0}\right|+2 \alpha,
$$

and so ||$x x_{0}|-| y y_{0}|| \leq 2 \alpha=\operatorname{dis}(R)$ for all $(x, y) \in R$.

Now we focus on trees. A metric space $X$ is called geodesic if for every pair of points $x, x^{\prime} \in X$ there is a geodesic segment $x x^{\prime} \subset X$ connecting the two points, i.e., the image of an isometric embedding of the interval $\left[0,\left|x x^{\prime}\right|\right]$ that sends 0 to $x$ and $\left|x x^{\prime}\right|$ to $x^{\prime}$. By a metric tree $X$ we mean a geodesic metric space with the property that for any triple $(x, y, z)$ of points in $X$ and any geodesic segments $x y, x z, y z$ connecting them, $x y \subset x z \cup y z$. Thus, geodesic triangles in $X$ are isometric to tripods, and geodesic segments are uniquely determined by their endpoints. For the next result, we need to sharpen the above assumption that $\pi_{X}(R)$ spans $X$. We say that a subset $A$ of a metric space $X$ strictly spans $X$ if for every pair $\left(x, x^{\prime}\right) \in X \times X$ there exists an $a \in A$ such that $\left|x x^{\prime}\right|+\left|x^{\prime} a\right|=|x a|$.

Proposition 2.2. Suppose that $X$ is a metric tree and $Y$ is an injective metric space. If $R \subset X \times Y$ is a set with the property that $\pi_{X}(R)$ strictly spans $X$, there exists an extension $R \subset \bar{R} \subset X \times Y$ such that $\pi_{X}(\bar{R})=X$ and $\operatorname{dis}(\bar{R})=\operatorname{dis}(R)$. 
In particular, every $\varepsilon$-roughly isometric map $f: A \rightarrow Y$ defined on a set $A \subset X$ that strictly spans $X$ admits an $\varepsilon$-roughly isometric extension $\bar{f}: X \rightarrow$ $Y$.

Proof. It suffices to show that for every set $R \subset X \times Y$ with $\operatorname{dis}(R)<\infty$ and the property that $\pi_{X}(R)$ spans $X$ and for every $\bar{x} \in X$ there exists a point $\bar{y} \in Y$ such that

$$
\operatorname{dis}(R \cup\{(\bar{x}, \bar{y})\})=\operatorname{dis}(R) .
$$

As above, the general result then follows by an application of Zorn's lemma.

Thus let such $R$ and $\bar{x}$ be given. Put $\alpha:=\operatorname{dis}(R) / 2$. As in the proof of Proposition 2.1, there exists a point $y_{0} \in Y$ with the property that

$$
\left|y y_{0}\right| \leq|x \bar{x}|+\alpha
$$

for all $(x, y) \in R$. Let $S$ be the set of all $(x, y) \in R$ with $\left|y y_{0}\right|<|x \bar{x}|-\alpha$. If $S=\emptyset$, then ||$x \bar{x}|-| y y_{0}|| \leq \alpha \leq \operatorname{dis}(R)$ for all $(x, y) \in R$; in particular, $\bar{y}:=y_{0}$ has the desired property. Suppose now that $S \neq \emptyset$, and fix an arbitrary $\left(x_{1}, y_{1}\right) \in S$. Since $\pi_{X}(R)$ strictly spans $X$, there exists a pair $\left(x_{2}, y_{2}\right) \in R$ such that $\left|x_{1} \bar{x}\right|+\left|\bar{x} x_{2}\right|=\left|x_{1} x_{2}\right|$. Now choose $\bar{y} \in Y$ so that $\left|\bar{y} y_{0}\right| \leq \alpha$ and $\left|\bar{y} y_{2}\right| \leq\left|y_{0} y_{2}\right|-\alpha$. Note that $\left|y_{0} y_{2}\right| \leq\left|\bar{x} x_{2}\right|+\alpha$, so $\left|\bar{y} y_{2}\right| \leq\left|\bar{x} x_{2}\right|$. For all $(x, y) \in R$,

$$
|y \bar{y}| \leq\left|y y_{0}\right|+\left|\bar{y} y_{0}\right| \leq\left|y y_{0}\right|+\alpha \leq|x \bar{x}|+2 \alpha \text {. }
$$

To estimate $|\bar{y} y|$ from below, note first that if $(x, y) \in R \backslash S$, then

$$
|y \bar{y}| \geq\left|y y_{0}\right|-\left|\bar{y} y_{0}\right| \geq\left|y y_{0}\right|-\alpha \geq|x \bar{x}|-2 \alpha \text {. }
$$

Secondly, let $(x, y) \in S$. Consider the tripod $x x_{1} \cup x x_{2} \cup x_{1} x_{2}$, and note that $\bar{x} \in x_{1} x_{2}$. Since $(x, y),\left(x_{1}, y_{1}\right) \in S$, the strict inequality

$$
\left|x x_{1}\right| \leq\left|y y_{1}\right|+2 \alpha \leq\left|y y_{0}\right|+\left|y_{0} y_{1}\right|+2 \alpha<|x \bar{x}|+\left|\bar{x} x_{1}\right|
$$

holds, so $\bar{x} \notin x x_{1}$ and therefore $\bar{x} \in x x_{2}$. We conclude that

$$
|y \bar{y}| \geq\left|y y_{2}\right|-\left|\bar{y} y_{2}\right| \geq\left(\left|x x_{2}\right|-2 \alpha\right)-\left|\bar{x} x_{2}\right|=|x \bar{x}|-2 \alpha .
$$

This shows that ||$x \bar{x}|-| y \bar{y}|| \leq 2 \alpha=\operatorname{dis}(R)$ for all $(x, y) \in R$.

The following example shows that Proposition 2.2 is no longer true in general if the word "strictly" is omitted.

Example 2.3. Let $X$ be the interval $[0,2]$, and put $x_{0}:=0$ and $x_{n}:=2-2^{-n}$ for all integers $n \geq 1$. The set $A:=\left\{x_{0}, x_{1}, \ldots\right\}$ spans $X$, but $A$ does not strictly span $X$ because $2 \notin A$. Let $Y$ be the simplicial metric tree with a single interior vertex $y_{1}$ and the countably many edges $y_{0} y_{1}$ and $y_{1} y_{n}$ for $n=2,3, \ldots$, where $\left|y_{0} y_{1}\right|=2^{-1}$ and $\left|y_{1} y_{n}\right|=2^{-1}-2^{-n}$. Note that $Y$ is complete, hence injective. The map $f: A \rightarrow Y$ defined by $f\left(x_{n}\right):=y_{n}$ for $n=0,1,2, \ldots$ is 1 -roughly isometric, as is easily checked. Since there is no pair of points at distance one in $Y, f$ does not admit a 1-roughly isometric extension $\bar{f}: X \rightarrow Y$.

However, the following holds. 
Lemma 2.4. Let $X$ be a metric tree, and suppose that $A \subset X$ is a set that spans $X$. Then there exists a dense subtree $\Sigma \subset X$ such that $A \subset \Sigma$ and $A$ strictly spans $\Sigma$.

Proof. Let $\Sigma$ be the union of all geodesic segments with both endpoints in $A$. Since $X$ is a metric tree, it is easily seen that for every pair of points $x, x^{\prime} \in \Sigma$, the geodesic segment $x x^{\prime}$ in $X$ is part of a geodesic segment $a a^{\prime}$ with $a, a^{\prime} \in A$. In particular, $\Sigma$ is a geodesic subspace of $X$, hence a metric tree, and $A$ strictly spans $\Sigma$. It remains to show that $\Sigma$ is dense in $X$. Let $x \in X$. Fix an arbitrary $a \in A$. Since $A$ spans $X$, for every $\varepsilon>0$ there is an $a_{\varepsilon} \in A$ so that $|a x|+\left|x a_{\varepsilon}\right| \leq\left|a a_{\varepsilon}\right|+\varepsilon$. Consider the geodesic segment $a a_{\varepsilon}$. Let $x_{\varepsilon}$ be the point on $a a_{\varepsilon}$ nearest to $x$. Then

$$
2\left|x x_{\varepsilon}\right|=|a x|+\left|x a_{\varepsilon}\right|-\left|a a_{\varepsilon}\right| \leq \varepsilon .
$$

Since $\varepsilon>0$ was arbitrary and $x_{\varepsilon} \in \Sigma, x$ lies in the closure of $\Sigma$.

3. Gromov-Hausdorff distance estimates. In this section we prove the results stated in the introduction. First we recall the definition of the GromovHausdorff distance. Let $\left(Z, d^{Z}\right)$ be a metric space. The usual Hausdorff distance $d_{\mathrm{H}}^{Z}(X, Y)$ of two subsets $X, Y$ of $Z$ is the infimum of all $\rho>0$ such that $X$ is contained in the (open) $\rho$-neighborhood of $Y$ and, vice versa, $Y$ lies in the $\rho$-neighborhood of $X$. More generally, if $X$ and $Y$ are two metric spaces, their Gromov-Hausdorff distance $d_{\mathrm{GH}}(X, Y)$ is defined as the infimum of all $\rho>0$ for which there exist a metric space $\left(Z, d^{Z}\right)$ and isometric copies $X^{\prime}, Y^{\prime} \subset Z$ of $X$ and $Y$, respectively, such that $d_{H}^{Z}\left(X^{\prime}, Y^{\prime}\right)<\rho$. The distance is always finite if $X$ and $Y$ are bounded, and for general metric spaces $X_{1}, X_{2}, X_{3}$ the triangle inequality $d_{\mathrm{GH}}\left(X_{1}, X_{2}\right)+d_{\mathrm{GH}}\left(X_{2}, X_{3}\right) \geq d_{\mathrm{GH}}\left(X_{1}, X_{3}\right)$ holds. Furthermore, $d_{\mathrm{GH}}$ induces an honest metric on the set of isometry classes of compact metric spaces.

The Gromov-Hausdorff distance of two metric spaces $X, Y$ may alternatively be characterized as follows. A correspondence $R$ between $X$ and $Y$ is a subset of $X \times Y$ such that the projections $\pi_{X}: X \times Y \rightarrow X$ and $\pi_{Y}: X \times Y \rightarrow Y$ are surjective when restricted to $R$. Then

$$
d_{\mathrm{GH}}(X, Y)=\frac{1}{2} \inf _{R} \operatorname{dis}(R),
$$

where the infimum is taken over all correspondences $R$ between $X$ and $Y$ (see [3, Theorem 7.3.25]). In view of this characterization, the following two theorems are now easy consequences of the results in the previous section.

Theorem 3.1. Suppose that $X, Y$ are two injective metric spaces, $A \subset X$ is a set that spans $X$, and $B \subset Y$ is a set that spans $Y$. Then

$$
d_{\mathrm{GH}}(X, Y) \leq 2 d_{\mathrm{GH}}(A, B) .
$$

Proof. Suppose that $R \subset A \times B$ is a correspondence between $A$ and $B$ with $\alpha:=\operatorname{dis}(R) / 2<\infty$. By Proposition 2.1, there is an extension $R \subset R_{1} \subset X \times Y$ such that $\pi_{X}\left(R_{1}\right)$ is an $\alpha$-net in $X$ and $\operatorname{dis}\left(R_{1}\right)=\operatorname{dis}(R)$, and there is a further extension $R_{1} \subset R_{2} \subset X \times Y$ such that $\pi_{Y}\left(R_{2}\right)$ is an $\alpha$-net in $Y$ and 
$\operatorname{dis}\left(R_{2}\right)=\operatorname{dis}\left(R_{1}\right)$. It is then easy to see how to extend $R_{2}$ to a correspondence $\bar{R}$ between $X$ and $Y$ so that $\operatorname{dis}(\bar{R}) \leq \operatorname{dis}\left(R_{2}\right)+2 \alpha=2 \operatorname{dis}(R)$. Hence,

$$
d_{\mathrm{GH}}(X, Y) \leq \frac{1}{2} \operatorname{dis}(\bar{R}) \leq \operatorname{dis}(R)
$$

and taking the infimum over all correspondences $R$ between $A$ and $B$ with finite distortion, we obtain the result.

For general metric $(\mathbb{R}$-)trees, the factor two in the above estimate may be dispensed with.

Theorem 3.2. Suppose that $X, Y$ are two metric trees, $A \subset X$ is a set that spans $X$, and $B \subset Y$ is a set that spans $Y$. Then

$$
d_{\mathrm{GH}}(X, Y) \leq d_{\mathrm{GH}}(A, B)
$$

Proof. Note that the completions $\bar{X}, \bar{Y}$ of $X, Y$ satisfy $d_{\mathrm{GH}}(\bar{X}, \bar{Y})=d_{\mathrm{GH}}(X, Y)$, and $A, B$ span $\bar{X}, \bar{Y}$, respectively. We thus assume, without loss of generality, that the metric trees $X, Y$ are complete, hence injective. Let $R \subset A \times B$ be a correspondence between $A$ and $B$. By Lemma 2.4, $A$ strictly spans a tree $X^{\prime} \supset A$ that is dense in $X$. Hence, by Proposition 2.2 , there is an extension $R \subset R_{1} \subset X^{\prime} \times Y$ such that $\pi_{X^{\prime}}\left(R_{1}\right)=X^{\prime}$ and $\operatorname{dis}\left(R_{1}\right)=\operatorname{dis}(R)$. We have $B \subset B^{\prime}:=\pi_{Y}\left(R_{1}\right)$, and so $B^{\prime}$ also spans $Y$. Again, $B^{\prime}$ strictly spans a tree $Y^{\prime} \supset B^{\prime}$ that is dense in $Y$, and there is an extension $R_{1} \subset R_{2} \subset X \times Y^{\prime}$ such that $\pi_{Y^{\prime}}\left(R_{2}\right)=Y^{\prime}$ and $\operatorname{dis}\left(R_{2}\right)=\operatorname{dis}\left(R_{1}\right)$. Since $\pi_{X}\left(R_{2}\right) \supset X^{\prime}$ is dense in $X$ and $Y^{\prime}$ is dense in $Y$, we obtain that

$$
d_{\mathrm{GH}}(X, Y)=d_{\mathrm{GH}}\left(\pi_{X}\left(R_{2}\right), Y^{\prime}\right) \leq \frac{1}{2} \operatorname{dis}\left(R_{2}\right)=\frac{1}{2} \operatorname{dis}(R) .
$$

As this holds for all correspondences $R$ between $A$ and $B$, this gives the result.

Next, in order to relate these results to the discussion in the introduction, we recall Isbell's explicit construction of the injective hull $\mathrm{E}(X)$ of a metric space $X$. We denote by $\mathbb{R}^{X}$ the vector space of all real functions on $X$. As a set, $\mathrm{E}(X)$ is defined as

$$
\mathrm{E}(X):=\left\{f \in \mathbb{R}^{X}: f(x)=\sup _{y \in X}(|x y|-f(y)) \text { for all } x \in X\right\},
$$

the set of the so-called extremal functions on $X$. For every $z \in X$, the distance function $d_{z}$, defined by $d_{z}(x):=|x z|$ for $x \in X$, belongs to $\mathrm{E}(X)$. In general, for every $f \in \mathrm{E}(X)$ and $z \in X$, the inequalities

$$
d_{z}-f(z) \leq f \leq d_{z}+f(z)
$$

hold, and it follows that $\left\|f-d_{z}\right\|_{\infty}:=\sup \left|f-d_{z}\right|=f(z)$. In particular, $\|f-g\|_{\infty}$ is finite for every pair of functions $f, g \in \mathrm{E}(X)$, and this equips $\mathrm{E}(X)$ with a metric. The map e: $X \rightarrow \mathrm{E}(X)$ that takes $x$ to $d_{x}$ is then a canonical isometric embedding of $X$ into $\mathrm{E}(X)$, as $\left\|d_{x}-d_{y}\right\|_{\infty}=|x y|$ for all $x, y \in X$. Isbell proved that $(\mathrm{e}, \mathrm{E}(X))$ is indeed an injective hull of $X$, i.e., $\mathrm{E}(X)$ is an injective metric space, and $(\mathrm{e}, \mathrm{E}(X))$ is a minimal such extension of $X$ in that no proper subspace of $\mathrm{E}(X)$ containing $\mathrm{e}(X)$ is injective. Furthermore, if $(i, Y)$ is another injective hull of $X$, then there exists a unique isometry 
$I: \mathrm{E}(X) \rightarrow Y$ with the property that $I \circ \mathrm{e}=i$. The following result explains how injective hulls are related to spanning subsets of (injective) metric spaces, in the sense of this paper.

Proposition 3.3. (1) For every metric space $A$, the image $\mathrm{e}(A)$ of the canonical isometric embedding e: $A \rightarrow \mathrm{E}(A)$ spans $\mathrm{E}(A)$.

(2) If $X$ is an injective metric space and $A \subset X$ is a set that spans $X$, then $X$ is isometric to $\mathrm{E}(A)$ via the map that sends $x \in X$ to the restricted distance function $\left.d_{x}\right|_{A}$.

Proof. For (1), let a pair $(f, g)$ of elements of $\mathrm{E}(A)$ be given, and let $\varepsilon>0$. There exists either a point $b \in A$ such that $\|f-g\|_{\infty} \leq f(b)-g(b)+\varepsilon / 2$ or a point $a \in A$ such that $\|f-g\|_{\infty} \leq g(a)-f(a)+\varepsilon / 2$. Then, by the definition of $\mathrm{E}(A)$, we may choose $a \in A$ with $f(b) \leq|a b|-f(a)+\varepsilon / 2$ in the first case and $b \in A$ with $g(a) \leq|a b|-g(b)+\varepsilon / 2$ in the second. In either case, this gives

$$
\|f-g\|_{\infty} \leq|a b|-f(a)-g(b)+\varepsilon .
$$

Since $|a b|-f(a) \leq f(b)=\left\|f-d_{b}\right\|_{\infty}$ and $g(b)=\left\|g-d_{b}\right\|_{\infty}$, we obtain that $\|f-g\|_{\infty} \leq\left\|f-d_{b}\right\|_{\infty}-\left\|g-d_{b}\right\|_{\infty}+\varepsilon$. As $d_{b}=\mathrm{e}(b) \in \mathrm{e}(A)$, this shows the claim.

For the proof of (2), let $x, y \in X$. Since $A$ spans $X$, we have first that for every $a \in A, d_{x}(a)=\sup _{b \in A}\left(|a b|-d_{x}(b)\right)$, so $\left.d_{x}\right|_{A} \in \mathrm{E}(A)$. Secondly, $|x y|=\sup _{a \in A}(|a x|-|a y|)$, which implies that the inequality

$$
\left\|\left.d_{x}\right|_{A}-\left.d_{y}\right|_{A}\right\|_{\infty}=\sup _{a \in A}|| a x|-| a y|| \leq|x y|
$$

is in fact an equality. Hence, the map that takes $x$ to $\left.d_{x}\right|_{A}$ is an isometric embedding of $X$ into $\mathrm{E}(A)$. Since $X$ is injective, so is the image of this map. Because no proper subspace of $\mathrm{E}(A)$ containing $\mathrm{e}(A)$ is injective, the image agrees with $\mathrm{E}(A)$.

In view of Proposition 3.3, Theorem 3.1 is equivalent to saying that for any metric spaces $A$ and $B$,

$$
d_{\mathrm{GH}}(\mathrm{E}(A), \mathrm{E}(B)) \leq 2 d_{\mathrm{GH}}(A, B),
$$

as stated in the introduction. We now show that the factor two is optimal.

Example 3.4. First we show that if $f: \mathbb{R} \times[0,4] \rightarrow \mathbb{R}$ is an $\varepsilon$-roughly isometric map, where $\mathbb{R} \times[0,4] \subset \mathbb{R}^{2}$ is endowed with the $l_{1}$ metric, then $\varepsilon \geq 4$. For any integer $n \geq 1$, consider the subset

$$
Z_{n}:=(\{0,8, \ldots, 8 n\} \times\{0\}) \cup(\{4,12, \ldots, 8 n-4\} \times\{4\})
$$

of $\mathbb{R} \times[0,4]$ of cardinality $2 n+1$. Note that, with respect to the $l_{1}$ distance, distinct points in $Z_{n}$ are at distance at least eight from each other, and the diameter of $Z_{n}$ equals $8 n$. Let $\left\{z_{1}, z_{2}, \ldots, z_{2 n+1}\right\}$ be an enumeration of $Z_{n}$ so that $f\left(z_{1}\right) \leq f\left(z_{2}\right) \leq \cdots \leq f\left(z_{2 n+1}\right)$. We have $f\left(z_{i+1}\right)-f\left(z_{i}\right) \geq\left\|z_{i+1}-z_{i}\right\|_{1}-$ $\varepsilon \geq 8-\varepsilon$, hence taking the sum from $i=1$ to $2 n$, we obtain $f\left(z_{2 n+1}\right)-f\left(z_{1}\right) \geq$ $2 n(8-\varepsilon)$. On the other hand, $f\left(z_{2 n+1}\right)-f\left(z_{1}\right) \leq \operatorname{diam}\left(Z_{n}\right)+\varepsilon=8 n+\varepsilon$. It follows that $\varepsilon \geq 8 n /(2 n+1)$. This holds for any $n \geq 1$, thus $\varepsilon \geq 4$. 
Now, for any $N>0$, consider the two metric spaces $A=\left\{a_{1}, \ldots, a_{4}\right\}$ and $B=\left\{b_{1}, \ldots, b_{4}\right\}$, where $\left|a_{1} a_{2}\right|=\left|a_{3} a_{4}\right|=4,\left|a_{1} a_{3}\right|=\left|a_{2} a_{4}\right|=N,\left|a_{1} a_{4}\right|=$ $\left|a_{2} a_{3}\right|=N+4,\left|b_{1} b_{2}\right|=\left|b_{3} b_{4}\right|=2$, and $\left|b_{i} b_{j}\right|=N+2(i \neq j)$ otherwise. The correspondence $\left\{\left(a_{1}, b_{1}\right), \ldots,\left(a_{4}, b_{4}\right)\right\}$ has distortion two, and since $\operatorname{diam}(A)=$ $\operatorname{diam}(B)+2$ there is no correspondence between $A$ and $B$ with distortion less than two. So $d_{\mathrm{GH}}(A, B)=1$. The injective hull $\mathrm{E}(A)$ is isometric to $[0, N] \times[0,4] \subset \mathbb{R}^{2}$ with the $l_{1}$ distance, and $\mathrm{E}(B)$ is a metric tree with a central edge of length $N$ and two edges of length one attached at each of its endpoints (like the tree $Y$ in Fig. 1). Let $\varepsilon_{0}<4$ be given. If $N$ is chosen big enough, depending on $\varepsilon_{0}$, essentially the same argument as above shows that there is no $\varepsilon$-roughly isometric map $f: \mathrm{E}(A) \rightarrow \mathrm{E}(B)$ with $\varepsilon<\varepsilon_{0}$. In particular, every correspondence between $\mathrm{E}(A)$ and $\mathrm{E}(B)$ has distortion at least $\varepsilon_{0} / 2$. In other words, for every $\delta_{0}<2$ we find a pair of four-point metric spaces $A, B$ so that $\mathrm{E}(A)$ is two-dimensional, $\mathrm{E}(B)$ is a metric tree, $d_{\mathrm{GH}}(A, B)=1$, and $d_{\mathrm{GH}}(\mathrm{E}(A), \mathrm{E}(B)) \geq \delta_{0}$.

\section{References}

[1] J. Adamek, H. Herrlich, And G. E. Strecker, Abstract and concrete categories: the joy of cats, Reprint of the 1990 original [Wiley], Repr. Theory Appl. Categ. No. 17 (2006), 1-507.

[2] N. Aronszajn and P. Panitchpakdi, Extension of uniformly continuous transformations and hyperconvex metric spaces, Pacific J. Math. 6 (1956), 405439.

[3] D. Burago, Y. Burago, and S. Ivanov, A Course in Metric Geometry, AMS, 2001.

[4] S. Buyalo And V. Schroeder, Elements of asymptotic geometry, EMS Monographs in Mathematics, 2007.

[5] A. Dress, Trees, tight extensions of metric spaces, and the cohomological dimension of certain groups: a note on combinatorial properties of metric spaces, Adv. in Math. 53 (1984), 321-402.

[6] A. Dress, K. T. Huber, And V. Moulton, An explicit computation of the injective hull of certain finite metric spaces in terms of their associated Buneman complex, Adv. in Math. 168 (2002), 1-28.

[7] A. Dress, V. Moulton, And W. Terhalle, T-theory: an overview, Europ. J. Combinatorics 17 (1996), 161-175.

[8] J. R. Isbell, Six theorems about injective metric spaces, Comment. Math. Helv. 39 (1964), 65-76.

[9] U. LANG, Injective hulls of certain discrete metric spaces and groups, J. Topol. Anal., doi:10.1142/S1793525313500118.

[10] A. MoezzI, The Injective Hull of Hyperbolic Groups, Dissertation ETH Zurich, No. 18860, 2010. 
URS LANG AND Mä̈L PAVÓN

Department of Mathematics, ETH Zurich, 8092 Zurich,

Switzerland

e-mail: lang@math.ethz.ch

MAËL PAVÓN

e-mail: mael.pavon@math.ethz.ch

Roger Züst

Département de Mathématiques,

Université de Fribourg,

1700 Fribourg,

Switzerland

e-mail: roger.zuest@unifr.ch

Received: 17 April 2013 